\title{
La trajectoire des symptômes dépressifs de l'enfance à l'adolescence et les cibles d'intervention préventive
}

\author{
A trajetória dos sintomas depressivos da infância \\ para a adolescência e objetivos da intervenção preventiva
}

Diane Marcotte ${ }^{1}$

Annie Lemieux ${ }^{1}$
${ }^{1}$ Département de Psychologie, Université du Québec à Montréal. 8888 succ. Centre-Ville Montréal H3C 3P8 Québec Canada. marcotte.diane@uqam.ca
Résumé Cette étude longitudinale s’intéresse à la trajectoire développementale des symptômes de dépression sur une période de cinq ans entre l'enfance et l'adolescence ainsi qu'à ses facteurs associés. À partir de l'analyse de courbes de croissance latente (Latent Growth Model-LGM), les résultats indiquent que les symptômes de dépression augmentent entre l'âge de 11-12 ans et 15-16 ans chez les filles, mais non chez les garçons. Cependant, pour chacun des sexes, la variance de la pente de la trajectoire reflète l'hétérogénéité des sousgroupes, révélant que les trajectoires varient chez les individus. Parmi les facteurs mesurés au temps 1 de l'étude, ceux qui sont le plus étroitement associés à la variation longitudinale des symptômes dépressifs sont le soutien des amis pour les deux genres et l'âge pour les filles. Plusieurs facteurs sont également associés à la présence de dépression au temps initial. Les cibles d'intervention pour une prévention universelle de la dépression chez les jeunes sont ensuite discutées et un programme visant le développement de facteurs de protection chez les adolescents est présenté.

Les mots clé La dépression, L'enfance, L'adolescence, La prévention
Resumo Este estudo longitudinal examina a trajetória de desenvolvimento de sintomas depressivos da infância à adolescência e fatores associados, ao longo de um período de cinco anos. A partir da análise de curvas de crescimento latentes (Modelo de Crescimento Latente/LGM), os resultados indicam que o aumento de sintomas depressivos entre meninas nas idades de 11-12 anos e de 15-16 anos, o que não ocorre entre meninos. No entanto, em ambos os sexos, a variação da inclinação da trajectória reflete a heterogeneidade dos sub-grupos, revelando que as trajectórias variam entre os indivíduos. Entre os fatores medidos no tempo 1 do estudo aqueles que estão mais estreitamente associados com a mudança longitudinal dos sintomas depressivos são o apoio dos amigos, para ambos os sexos e a idade entre as meninas. Vários factores estão associados com a presença de depressão no tempo inicial. São discutidos objetivos de intervenção para a prevenção universal de depressão entre os jovens, assim como é apresentado um programa para o desenvolvimento de fatores de proteção entre adolescentes.

Palavras-chave Depressão, Infância, Adolescência, Prevenção 


\section{Introduction}

Les taux d'épisodes dépressifs majeurs sont faibles pendant l'enfance, soit entre $1 \%{ }^{1}$ et $3 \%^{2}$. L'apparition du premier épisode dépressif se situe entre 11 et 14 ans $^{3,4}$ et une augmentation marquée se produit entre l'âge de 11 et 15 ans pour atteindre un sommet au début de l'âge adulte, soit vers 21 à $26 \mathrm{ans}^{5,6}$. Alors que les taux sont similaires entre les genres ou plus élevés chez les garçons avant l'adolescence ${ }^{7}$, les filles présentent des taux deux à quatre fois plus élevés à partir de l’adolescence ${ }^{8}$. Ces taux élevés chez les filles apparaissent aux alentours de l'âge de 13 ans et sont associés au statut pubertaire davantage qu'à l'âge chronologique. Les taux augmentent sensiblement chez les deux genres mais l'augmentation est plus marquée chez les filles. Hankin, et al. ${ }^{9}$ ont mesuré la dépression à partir d'une entrevue diagnostique sur un interval de dix ans et leurs résultats suggèrent la présence de deux période d'augmentation, l'une entre 13 et 15 ans et la seconde entre 15 et 18 ans. Ces chercheurs observent une différence entre les genres pendant la période de 13-15 ans, mais cette différence devient davantage observable entre 15 et 18 ans. Puisque cette différence demeure présente pour le reste de la vie, il devient primordial d'étudier cette période développementale afin de comprendre les facteurs associés à cette augmentation ainsi qu'à l'émergence de cette différence entre les genres

Les résultats des études concernant les trajectoires des garçons et des filles en ce qui a trait aux syndromes et symptômes dépressifs sont moins consistants ${ }^{10}$; le premier étant le plus souvent mesuré par la proportion de participants qui atteignent un score de coupure sur une mesure auto-évaluative de la dépression alors que le deuxième réfère au niveau ou l'intensité des symptômes auto-rapportés. Par exemple, GonzálezTejera et al. ${ }^{11}$ n'ont observé aucune différence entre les genres pour le syndrome dépressif, une différence n'apparaissant seulement lorsque le nombre de symptômes était plus élevé. Dans une méta-analyse, Twenge et Nolen-Hoeksema ${ }^{12}$ rapportent que pendant l'adolescence, les symptômes dépressifs augmentent à partir de l'âge de 13 ans chez les filles mais non chez les garçons; chez ces derniers le niveau demeure relativement stable ou augmente à un rythme plus lent que chez les filles.

Quelques études longitudinales ont également examinél'existence de sous-groupes d'adolescents selon les trajectoires suivies pendant la transition de l'enfance à l'adolescence ${ }^{13,14}$. À titre d'exemple, dans l'étude de Dekker et al. ${ }^{13}$, les garçons qui suivent une trajectoire caractérisée par un niveau élevé de symptômes dépressifs pendant l'enfance et qui diminue ensuite significativement dès le début de l'adolescence présentent plus de probabilités de présenter un faible niveau de scolarité. Cette étude souffre cependant d'une lacune méthodologique importante puisque les symptômes de dépression ont été évalués par les parents uniquement. De même, Brendgen, et al. ${ }^{14}$, ont réalisé une étude auprès de jeunes âgés entre 11 et 14 ans et proposent quatre sous-groupes dont un présentant un faible niveau de dépression (50\% des participants), un moyennement déprimé (30\%), un très déprimé $(10 \%)$ et un sous-groupe dont les symptômes augmentent entre 11 et 14 ans (10\%). Cette étude ne couvre cependant qu'une courte période de temps entre 11 et 14 ans, alors que nous savons que l'augmentation de la dépression apparaît particulièrement entre 13-15 ans. De plus, les analyses ont été réalisées sur l'échantillon complet sans s'attarder aux différences entre les garçons et les filles.

Encore plus rares sont les études qui ont tenté d'identifier les facteurs personnels, familiaux ou scolaires associés aux trajectoires des symptômes dépressifs chez les adolescents. Dans une étude récente de Wang et al. ${ }^{15}$, réalisée à partir d'un large échantillon, l'influence de variables scolaires et familiales sur les trajectoires des symptômes dépressifs et des problèmes de comportement a été explorée. Les chercheurs rapportent un déclin des symptômes dépressifs entre 13 et 18 ans. Les garçons présentent un niveau de symptômes plus faible que les filles à 13 ans et la diminution des symptômes au fil du temps est plus importante chez eux que chez les filles. Les résultats démontrent qu'une relation positive avec l'enseignant, de même qu'un faible niveau de conflits avec les parents à l'âge de 13 ans sont tous deux associés à un niveau initial plus faible de symptômes dépressifs ainsi qu'à une diminution plus rapide des symptômes de dépression entre 13 et 18 ans. Cette étude débute cependant à l'âge de 13 ans et ainsi ne couvre pas la période de transition de l'enfance à l'adolescence.

Ainsi, malgré que plusieurs études rapportent l'augmentation des symptômes dépressifs, ceci particulièrement chez les filles, entre l'enfance et l'adolescence, la trajectoire distincte suivie par chaque sexe demeure à examiner plus précisément. À ce jour, encore très peu d'étude ont considéré les genres séparément. De plus, l'intervalle de temps mesuré s'avère insuffisamment long pour couvrir la période de transition entre l'en- 
fance et l'adolescence. Également, certaines études ont été réalisées auprès de populations à risques plutôt qu'une population générale. Or, il est reconnu que la période de transition entre l'enfance et l'adolescence comporte de nombreux défis et constitue une période à risque, notamment parce que cette transition développementale se produit en synchronie avec une autre transition importante, celle-ci institutionnelle que représente la transition de l'école primaire vers l'école secondaire. Dans une perspective développementale de la psychopathologie, il est pertinent de s'intéresser à l'identification des facteurs qui influencent la vulnérabilité à la dépression chez les adolescents. Les défis normatifs de l'adolescence, tel que l'arrivée de la puberté, la transition primaire-secondaire et le développement de l'autonomie face aux parents peuvent influencer directement le risque de dépression ou interagir avec des caractéristiques individuelles ou encore, des stress non normatifs, pour augmenter la vulnérabilité. Dans cette étude, un ensemble de facteurs personnels, familiaux et scolaires ont été étudiés en relation avec les trajectoires des symptômes dépressifs des garçons et des filles à partir de 11-12 ans, soit avant la transition primairesecondaire, jusqu'à l'âge de 15-16 ans.

\section{Objectifs de l'étude}

Le premier objectif de cette étude est d'examiner les trajectoires des symptômes dépressifs chez les garçons et les filles de 11-12 ans à 15-16 ans. Le deuxième objectif vise à identifier les facteurs personnels, scolaires et familiaux qui influencent l'intensité des symptômes dépressifs initiaux, soit à l'âge de 11-12 ans, ainsi que la variation variation dans l'évolution de la trajectoire longitudinale de ces derniers entre 11-12 ans et 15-16 ans.

\section{Methode}

\section{Participants et procédures}

Les données constituent les cinq premières années d'une étude longitudinale d'une durée de neuf ans (2003-2012) sur l'émergence de la dépression pendant les transitions scolaires. L'échantillon comprend 499 adolescents et adolescentes de $6^{e}$ année, soit la dernière année avant le passage vers l'école secondaire, provenant de 12 écoles primaires québécoises au temps initial. Seuls les élèves qui n'atteignent pas un niveau de troisième année en lecture ont été exclus. L'âge moyen des adolescents est de 11,26 ans (écarttype; $\hat{\mathrm{E}}-\mathrm{T}=0,481$ ) et celui des adolescentes est de $11,19$ ans (É-T $=0,421)$. L'échantillon est composé à $52,5 \%$ de garçons $(\mathrm{n}=262)$ et $47,5 \%$ de filles $(n=237)$. Parmi eux, $63,9 \%$ des jeunes vivaient avec leurs parents au temps initial, 17,5\% vivaient en garde partagée, $11,2 \%$ vivaient avec un parent monoparental, $3,8 \%$ vivaient en famille recomposée et les derniers $3,6 \%$ des jeunes vivaient en famille d'accueil ou autres. Les participants ont affirmés vivre au sein d'une famille comportant en moyenne au temps initial 1,65 enfants ( $(\hat{\mathrm{E}}-\mathrm{T}=1,15)$. La grande majorité des jeunes se perçoit dans la moyenne ou au-dessus $(93,9 \%)$ quant à leur rendement scolaire. Les indices de défavorisation des deux commissions scolaires participantes indiquent que les participants sont issus de la classe moyenne à moyenne-élevée ${ }^{16}$. Les questionnaires ont été administrés à chaque automne dans le cadre d'un cours régulier par deux assistants de recherche formés. Les enseignants complétaient également les mesures. À la fin de chaque année, les résultats au sommaire en français et en mathématiques ont été recueillis à partir du dossier scolaire de l'élève. Compte tenu de la forte corrélation entre ces deux variables, une moyenne a été calculée pour chaque participant, afin d'obtenir la variable rendement scolaire réel.

\section{Instruments de mesure}

Plusieurs mesures auto-évaluatives ont été complétées par les participants. Les symptômes de dépression ont été mesurés à l'aide de l'Inventaire de dépression de Beck, $2^{e}$ édition (IBD-II) ${ }^{17}$. Cette mesure auto-évaluative comprend 21 énoncés, offrant chacun quatre choix de réponses correspondants à une intensité croissante d'un symptôme noté de 0 à 3 . Le résultat total représente la sévérité des symptômes dépressifs chez un individu. Les attitudes dysfonctionnelles ont été mesurées par l'Échelle des Attitudes Dysfonctionnelles $(\mathrm{EAD})^{18,19}$. L'instrument comprend 24 énoncés répartis en trois sous-échelles (réussite, dépendance et autocontrôle). Chaque énoncé est associé à une échelle de réponse de type Likert en sept points allant de complètement d'accord à complètement en désaccord. Le statut pubertaire a été mesuré à l'aide de l'Échelle de développement pubertaire ${ }^{20,21}$. Cette mesure auto révélée se base, chez les garçons, sur la poussée des poils et la mue de la voix et, chez les filles, sur la poussée des poils, le développement de la poitrine et les menstruations afin 
d'évaluer le niveau de développement pubertaire du participant. L'image corporelle a été mesurée à l'aide d'une sous-échelle du Self Perception Profile for Adolescent ${ }^{22}$ qui évalue la perception de soi relative à l'apparence physique. La perception du soutien des amis a été mesurée par le Perceived Social Support-Friends (PSS-A) ${ }^{23}$. Cet instrument vise à évaluer si les besoins de soutien, d'information et de rétroaction sont comblés par les amis. Il comprend 20 énoncés associés à une échelle de réponse de type Likert en sept points. Le Questionnaire d'agression et de victimisation ${ }^{24}$ mesure par le biais de 15 questions, divers aspects reliées à l'intimidation et à la victimisation. Le questionnaire Échelle de l'environnement familial'25 est composé de 45 items qui mesurent les caractéristiques sociales et environnementales de la famille. Le choix de réponse est vrai ou faux. Cinq sous-échelles évaluent les dimensions suivantes: (a) cohésion, (b) expression des sentiments, (c) conflits, (d) organisation, et (e) contrôle. Pour sa part, l'Échelle de l'environnement de classe ${ }^{26}$ permet d'évaluer le climat social de la classe en mesurant et décrivant les relations élève/enseignant et élève/élève ainsi que le type de structure organisationnelle de la classe. Sa version abrégée comprend 36 énoncés répartis également sur neuf sous-échelles : 1) engagement, 2) affiliation, 3) soutien de l'enseignant, 4) orientation vers le travail, 5) compétition, 6) ordre et l'organisation, 7) clarté des règlements, 8) contrôle de l'enseignant et 9) innovation. Les réponses sont de types vrai ou faux. Finalement, un questionnaire de dépistage d'élèves à risque de décrochage scolaire a été administré2 ${ }^{2}$.

Les enseignants ont complété le Behavior Assessment System for Children-Teacher Report (BASC-TR) de Reynolds et Kamphaus ${ }^{28,29}$ qui permet d'évaluer les aspects adaptatifs et négatifs du comportement des adolescents à l'école. Il s'apparente au Achenbach $(r=0.93)$, mais présente des qualités psychométriques supérieures en milieu scolaire ${ }^{30}$. Dans la présente étude, trois dimensions du comportement ont été évaluées, soit les problèmes d'attention, les troubles des conduites et les habiletés sociales.

\section{Stratégies d'analyses}

Les données ont été analysées à partir d'analyses de trajectoires de croissance latentes (Latent Growth Modeling - LGM). Les LGM font partie des analyses de modèles d'équations structurales: La particularité de cette technique d'analyse statistique est qu'elle permet de calculer la variabilité intrasujet et intersujet simultanément, ce qui si- gnifie que l'on tient compte de la trajectoire individuelle de chaque sujet ainsi que de la trajectoire moyenne du groupe. Ainsi, le but de l'analyse est d'estimer une trajectoire qui représente le mieux les observations répétées selon le temps de chaque cas. Le logiciel Mplus (Muthen et Muthen 5.1) permet de réaliser ces analyses.

Les courbes de croissance ou trajectoires obtenues pour chaque sexe ont été examinées entre les temps 1 et 5 de l'étude, soit de la $6^{e}$ année (1112 ans) du primaire à la $4^{e}$ secondaire (15-16 ans). L'étude possède un taux d'attrition de moins de $10 \%$ sur les cinq temps de mesure. Les analyses ont été effectuées en deux étapes. Premièrement, les modèles de base (unconditional model) ont été estimés pour le nombre de symptômes de dépression sur les cinq temps de mesure séparément pour les garçons et pour les filles. La deuxième étape de l'analyse a consisté en la construction d'un modèle final avec covariables (conditional model) mesurées au temps 1 de l'étude.

Chaque individu possède sa propre trajectoire et sa propre variabilité dans le temps, tout comme chaque groupe détient une trajectoire moyenne ainsi que sa propre variabilité. Cette variance influence la courbe de la trajectoire au niveau de l'intercepte (intercept), qui représente dans cette étude le statut initial du nombre de symptômes de dépression, et la pente (slope) qui traduit la trajectoire dans le temps du nombre de symptômes. Plus la variabilité de l'intercepte est grande, plus les individus divergent en ce qui a trait au statut initial (i.e. nombre de symptômes de dépression) de leur trajectoire. D'un autre côté, une variabilité importante de la pente signifient que les participants ont une évolution temporelle distincte.

Ces covariables sont ajoutées dans le modèle de base afin de déterminer lesquelles influencent la trajectoire sur l'intercepte, la pente et/ou le terme quadratique. Cette étape a été réalisée sur chacun des deux modèles de base (garçons et filles) afin de bien identifier les covariables influençant chacune des trajectoires. Pour chacun des deux modèles, seules les covariables significatives à moins de $10 \%$ sur l'intercepte, la pente et/ou le terme quadratique sont retenues pour le modèle final. Les modèles de base obtenus pour chaque sexe sont illustrés à la Figure 1 et le Tableaux 1 présentent les estimateurs des facteurs de croissance et les statistiques des modèles.

Les analyses ont été effectuées en deux étapes. Premièrement, les modèles de base (unconditional model) ont été estimés pour le nombre de symptômes de dépression sur les cinq temps de mesure séparément pour les garçons et pour les 


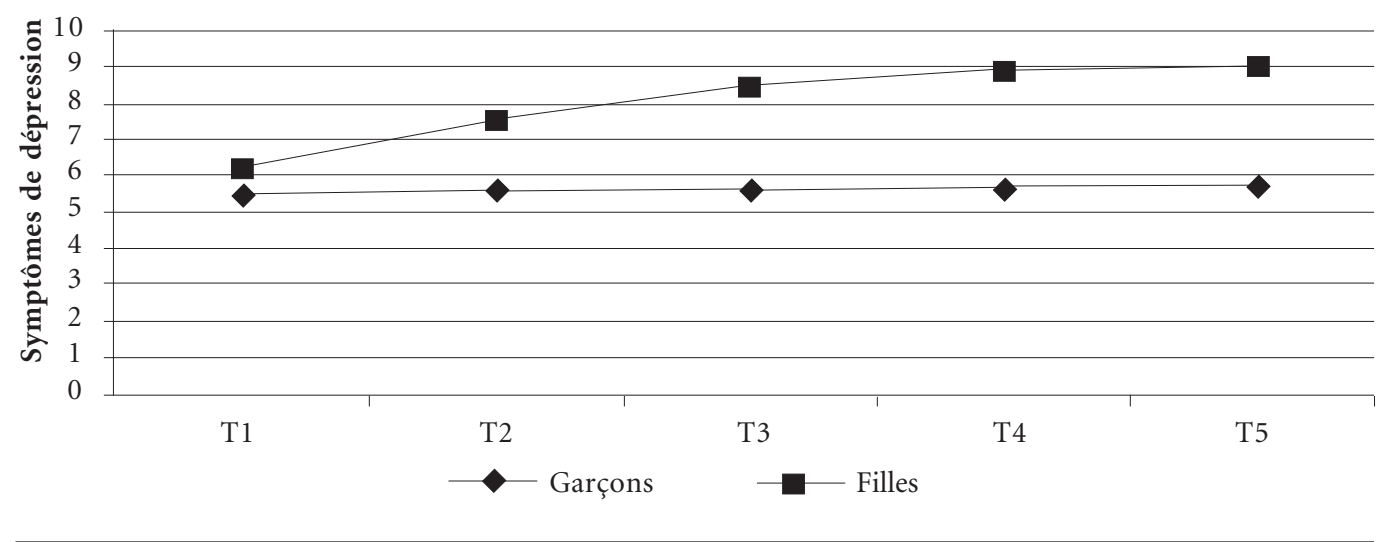

Figure 1. Trajectoires de base LGM des garçons et des filles.

Tableau 1. Paramètres du LGM de base chez les garçons et les filles.

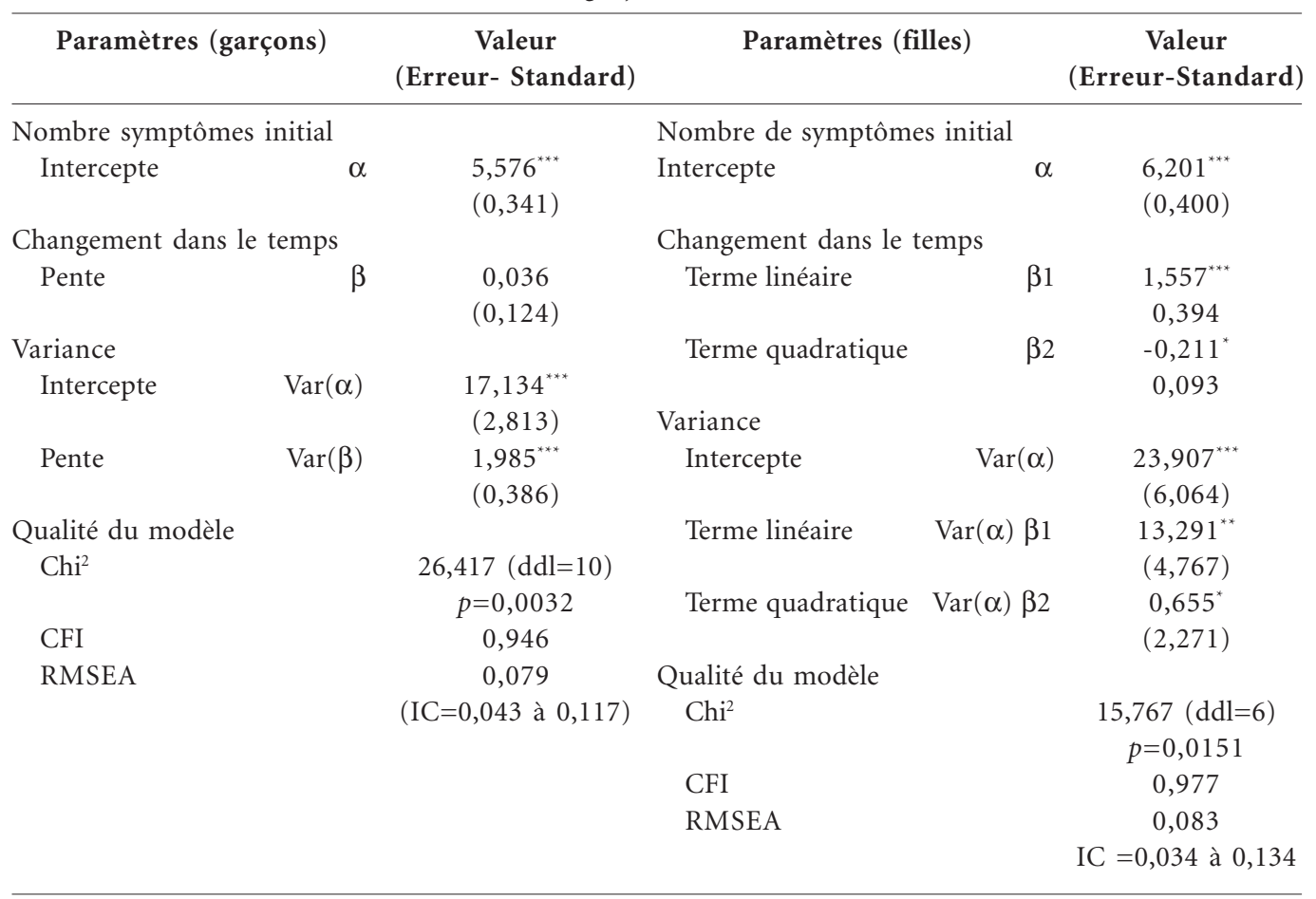

${ }^{*} p<0,05 \quad{ }^{* *} p<0,01 \quad{ }^{* * *} p<0,001$

filles. Ces modèles ont été estimés à l'aide des LGM à deux facteurs latents, soit l'intercepte et la pente. Le premier facteur décrit le statut initial du nombre de symptômes (moyenne de l'intercepte) et la variabilité des individus sur leur niveau initial du nombre de symptômes (variable de l'intercepte). L'intercepte de la trajectoire est cons- tant dans le temps, c'est pourquoi ces coefficients de saturation, factor loadings en anglais, sont fixés à 1 sur les cinq temps de mesures. Le second facteur de croissance des LGM décrit le sens du changement dans le temps ainsi que son taux (moyenne de la pente). Il permet aussi de détecter la présence de différentes trajectoires entre les indivi- 
dus (variance de la pente). Les coefficients de saturation de la pente peuvent être fixés sur les cinq temps afin de correspondre aux mesures du temps, ceci représente une trajectoire linéaire. Ils peuvent aussi être estimés par un modèle quadratique. Cette dernière option suppose la nonlinéarité de la trajectoire. Ces deux méthodes ont été testées dans cette analyse.

La deuxièmement étape de l'analyse sera la construction d'un modèle final avec covariables qui auront été judicieusement sélectionnées. Leurs choix reposeront sur des critères conceptuels et théoriques. Ces covariables seront ajoutées dans le modèle de base afin de déterminer lesquelles influencent la trajectoire sur l'intercepte et/ou la pente. Cette étape sera faite sur chacun des deux modèles de base (garçons et filles) afin de bien identifier les covariables influençant chacune des trajectoires. Pour chacun des deux modèles, seules les covariables significatives à moins de 5\% sur l'intercepte et/ou la pente seront retenues pour le modèle final (conditional model).

Afin de valider les modèles, les statistiques telles que le Root Mean Square Error of Approximation (RMSEA) ${ }^{31}$, le Comparative Fit Index $(\mathrm{CFI})^{32}$ et le $\mathrm{Chi}^{2}$ seront utilisées. Pour un bon modèle, Hu et Bentler ${ }^{33}$ suggèrent un RMSEA < 0,06 quoique ces auteurs jugent tout à fait acceptable un critère de 0,08 dans le cas d'échantillons relativement petits $(\mathrm{n}<500)$, comme c'est le cas ici. Aussi, ils proposent un CFI idéal $>0,960$ et, minimalement, de 0,900 . Quant à la valeur du $\mathrm{Chi}^{2}$, bien que très influencée par la taille de l'échantillon, elle doit idéalement être suffisamment petite pour ne pas atteindre le seuil de signification de $p<0,05^{34}$.

\section{Résultats}

Pour les garçons, le modèle linéaire estimé présente un intercepte significatif, $\alpha=5,576$ (erreurstandard $[\mathrm{E}-\mathrm{S}]=0,341 ; p<0,001)$ et une pente non significative, $\beta=0,036$ (E-S $=0,124 ; p=$ $0,769)$. Ainsi, les 262 adolescents ont un nombre de symptômes moyen initial de 5,576 sur l'échelle de Beck et leur trajectoire moyenne est stable sur les cinq temps de mesure. La variance de l'intercepte est significative $\operatorname{Var}(\alpha)=17,134$ (E-S $=$ $2,813 ; p<0,001)$, ce qui indique une grande variabilité dans le statut initial du nombre de symptômes. La variance de la pente est aussi très significative $\operatorname{Var}(\hat{\mathrm{a}})=1,985(\mathrm{E}-\mathrm{S}=0,386 ; p<0,001)$, ce qui indique aussi une grande variabilité dans le changement dans le temps chez les adolescents.
Donc, malgré que la trajectoire moyenne des adolescents soit stable, la variabilité de la pente permet de croire que certains d'entre eux diminuent et que d'autres augmentent leur nombre de symptômes dans le temps.

Pour les filles, le modèle estimé présente un intercepte significatif, $\alpha=6,201$ (erreur-standard $[\mathrm{E}-\mathrm{S}]=0,400 ; p<0,001)$, une pente significative, $\beta_{1}=1,557$ (E-S $\left.=0,394 ; p<0,001\right)$ et un terme quadratique aussi significatif, $\beta_{2}=-0,211$ (E-S = $0,093 ; p<0,05)$. Donc, les adolescentes ont un nombre de symptômes moyen initial de 6,201 sur l'échelle de Beck. Leur pente positive et significative indique une augmentation marquée dans le temps du nombre de symptômes sur l'échelle de Beck. Le terme quadratique négatif et significatif indique une amplitude négative de la trajectoire; c'est-à-dire qu'on observe une augmentation rapide du nombre de symptômes au début de la trajectoire suivie d'un ralentissement aux derniers temps de mesure. La variance de l'intercepte est significative $\operatorname{Var}(\alpha)=23,907$ (E-S $=$ $6,064 ; p<0,001)$, ce qui indique une grande variabilité dans le statut initial du nombre de symptômes. La variance de la pente est significative $\operatorname{Var}\left(\beta_{1}\right)=13,291(\mathrm{E}-\mathrm{S}=4,767 ; p<0,01)$, ce qui indique aussi une grande variabilité dans le changement dans le temps chez les adolescents. La variance du terme quadratique est aussi significative $\operatorname{Var}\left(\beta_{2}\right)=0,655$ (E-S $\left.=0,271 ; p<0,05\right)$, ce qui signifie une variabilité quant à l'amplitude de la trajectoire. Chez les filles également, les variances significatives permettent de croire que les filles n'ont pas toutes la même trajectoire dans le temps.

Ensuite, la deuxième étape des analyses a consisté en l'ajout des covariables au temps 1 de l'étude, ce qui se justifie par la présence de variances significatives à l'intérieur des modèles de base (interceptes, pentes et/ou termes quadratiques). Le modèle final des garçons compte cinq covariables trouvées significatives sur l'intercepte et/ou la pente, soit les conflits familiaux, l'image corporelle, le statut pubertaire, être victime d'intimidation et le soutien des amis. Parmi ces cinq covariables, quatre sont significatives sur l'intercepte. Donc, la présence de conflits familiaux $(0,578)$, un statut pubertaire plus avancé $(1,553)$ et le fait d'être victime d'intimidation $(2,858)$ sont des covariables liées positivement à l'intercepte; c'est-à-dire qu'elles prédisent plus de symptômes de dépression au temps initial, soit à 11-12 ans ( 6 e année) avant la transition primaire-secondaire. De plus, on observe que la perception de l'image corporelle $(-1,842)$ est liée négativement avec l'intercepte; c'est-à-dire que les garçons qui avaient 
une bonne image corporelle au temps initial de l'étude présentaient aussi moins de symptômes de dépression. Parmi ces quatre covariables, seul le soutien des amis $(0,459)$ est significativement (moins de 5\%) et positivement lié à la pente de la trajectoire de dépression; c'est-à-dire qu'on observe étonnamment une décélération moins marquée des symptômes de dépression au fil du temps lorsque le jeune rapportait un grand soutien amical avant la transition (Temps 1, 11-12 ans, $6^{\mathrm{e}}$ année). Le Tableau 2 présente le résumé du modèle final des garçons et la Figure 2 présentent les valeurs standardisées des covariables significatives à $5 \%$ ou moins.

Le modèle final avec co-variables des filles compte huit covariables trouvées significatives sur l'intercepte, la pente et/ou le terme quadratique, soit l'âge, la cohésion familiale, les conflits familiaux, l'image corporelle, le rendement en français, l'engagement scolaire, être victime d'intimidation et le soutien des amis. Parmi les huit covariables, cinq sont significatives à $5 \%$ sur l'intercepte. L'âge $(2,522)$, les conflits familiaux $(0,441)$ et être victime d'intimidation $(3,987)$ sont des covariables positivement liées à l'intercepte. Cela signifie qu'elles prédisent la présence d'un nombre élevé de symptômes de dépression au temps initial de la trajectoire, soit avant la transition. De leur côté, la cohésion familiale $(-0,547)$ et l'engagement scolaire $(-0,730)$ sont des covariables négativement liées à l'intercepte. L'image corporelle
$(-0,895)$ est aussi une covariable négativement liée à l'intercepte, mais, elle est marginalement significative. Toujours parmi les huit covariables,

Tableau 2. Paramètres du modèle final LGM chez les garçons.

\begin{tabular}{lrc}
\hline \multicolumn{1}{c}{ Paramètres } & Valeur & $\begin{array}{c}\text { Erreur } \\
\text { Standard }\end{array}$ \\
\hline Intercepte & $8,523^{* * *}$ & 2,332 \\
Conflits familiaux & $0,578^{* * *}$ & 0,139 \\
Image corporelle & $-1,842^{* * *}$ & 0,497 \\
Statut pubertaire & $1,553^{* *}$ & 0,467 \\
Victime d'intimidation & $2,858^{*}$ & 1,373 \\
Soutien des amis & $-0,501$ & 0,438 \\
Pente & $-2,056^{*}$ & 0,918 \\
Conflit & $-0,098^{\dagger}$ & 0,054 \\
Image corporelle & 0,322 & 0,206 \\
Statut pubertaire & $-0,329^{\dagger}$ & 0,180 \\
Victime d'intimidation & $-0,075$ & 0,539 \\
Soutien des amis & $0,459^{* *}$ & 0,172 \\
Variance & & \\
Intercepte & $10,701^{* * *}$ & 2,294 \\
Pente & $1,603^{* * *}$ & 0,360
\end{tabular}

Qualité du modèle

$\begin{array}{lc}\text { Chi }^{2} & 41,644(\mathrm{ddl}=25) \\ & p=0,0196 \\ \text { CFI } & 0,954 \\ \text { RMSEA } & 0,050\end{array}$

${ }^{\dagger} p<0,1{ }^{*} p<0,05{ }^{* *} p<0,01{ }^{* * *} p<0,001$

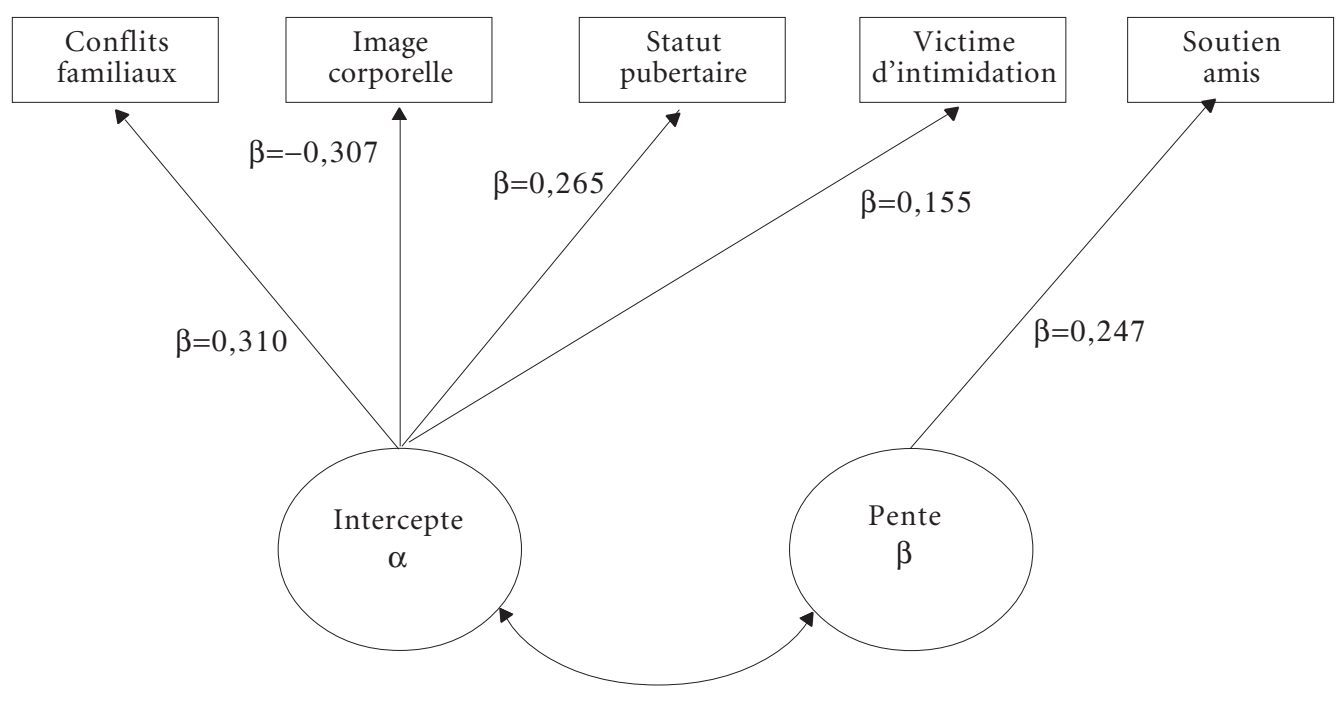

Figure 2. Description des effets (bêtas standardisés) des covariables significatives du modèle final des garçons sur l'intercepte et la pente. Tous les effets sont significatifs à $p<0,05$. 
Tableau 3. Paramètres du modèle final LGM chez les filles.

\begin{tabular}{|c|c|c|}
\hline Paramètres & Valeur & $\begin{array}{c}\text { Erreur } \\
\text { Standard }\end{array}$ \\
\hline Intercepte & $14,456^{* * *}$ & 4,130 \\
\hline Age & $2,522^{* *}$ & 0,831 \\
\hline Cohésion familiale & $-0,547^{* *}$ & 0,211 \\
\hline Conflits familiaux & $0,441^{*}$ & 0,186 \\
\hline Image corporelle & $-0,895^{\dagger}$ & 0,469 \\
\hline Rendement français & $-0,054$ & 0,041 \\
\hline Engagement scolaire & $-0,730^{*}$ & 0,317 \\
\hline Victime d'intimidation & $3,987^{* *}$ & 1,265 \\
\hline Soutien des amis & 0,011 & 0,523 \\
\hline Pente & $-4,806^{*}$ & 4,882 \\
\hline Age & $-2,104^{*}$ & 0,974 \\
\hline Cohésion familiale & $-0,043$ & 0,245 \\
\hline Conflits familiaux & $-0,085$ & 0,218 \\
\hline Image corporelle & $-0,907^{\dagger}$ & 0,544 \\
\hline Rendement français & $0,084^{\dagger}$ & 0,048 \\
\hline Engagement scolaire & $-0,325$ & 0,371 \\
\hline Victime d'intimidation & $-0,439$ & 1,502 \\
\hline Soutien des amis & $1,299^{*}$ & 0,614 \\
\hline Terme quadratique & 1,269 & 1,168 \\
\hline Age & 0,282 & 0,233 \\
\hline Cohésion familiale & 0,047 & 0,058 \\
\hline Conflits familiaux & 0,020 & 0,052 \\
\hline Image corporelle & 0,183 & 0,129 \\
\hline Rendement français & $-0,016$ & 0,011 \\
\hline Engagement scolaire & 0,093 & 0,088 \\
\hline Victime d'intimidation & $-0,006$ & 0,358 \\
\hline Soutien des amis & $-0,351^{*}$ & 0,148 \\
\hline \multicolumn{3}{|l|}{ Variance } \\
\hline Intercepte & $8,927^{\dagger}$ & 4,852 \\
\hline Pente & $9,768^{*}$ & 4,409 \\
\hline Terme quadratique & $0,540^{*}$ & 0,262 \\
\hline
\end{tabular}

Qualité du modèle

$\begin{array}{lc}\mathrm{Chi}^{2} & 41,305(\mathrm{ddl}=22) \\ & p=0,0076 \\ \text { CFI } & 0,964 \\ \text { RMSEA } & 0,061\end{array}$

${ }^{\dagger} p<0,1{ }^{*} p<0,05{ }^{* *} p<0,01 \quad{ }^{* * *} p<0,001$

deux ont été trouvées significatives sur la pente. L'âge $(-2,104)$ est lié négativement à la pente et le soutien des amis $(1,299)$ est lié positivement. Cela signifie que malgré que l'âge prédise plus de symptômes au temps initial, il semble que les filles plus âgées ne conservent pas cette avance au fil des cinq temps et les filles qui rapportaient avoir beaucoup de soutien de leurs amis au temps initial semblent développer plus de symptômes de dépression au fil du temps. Aussi, l'image corporelle $(-0,907)$ et le rendement en français $(0,084)$ sont des variables marginalement significatives sur la pente. La seule covariable trouvée significative sur le terme quadratique est de nouveau le soutien des amis $(-0,351)$; c'est-à-dire que malgré que les filles qui rapportaient avoir beaucoup de soutien amical au temps initial, soit en 11-12 ans ( $6^{e}$ année), ont développé plus de symptômes au fil du temps, cette augmentation freine avec le temps. Le Tableau 3 présente le résumé du modèle final chez les filles et la Figure 3 présente les valeurs standardisées des covariables significatives à $5 \%$ ou moins.

\section{Discussion des résultats et présentation du programme de prévention}

Cette étude a permis d'examiner en profondeur la trajectoire des symptômes dépressifs pendant la transition de l'enfance à l'adolescence et d'identifier les dimensions personnelles, familiales et scolaires associées à la présence de dépression pendant cette étape importante de la vie. Les variances significatives observées tant des interceptes que des pentes des courbes confirment que certains jeunes sont plus vulnérables face à la dépression et tous ne suivent pas la même trajectoire. Malgré que, vue dans son ensemble, la population des garçons ne présente pas de hausse de symptômes dépressifs lors de cette transition vers l'adolescence, ceux parmi eux qui vivent une puberté précoce et dont l'appréciation de leur image corporelle est négative seront plus à risque de présenter des symptômes de dépression avant même l'adolescence, soit dès l'âge de 11-12 ans ou $6^{\mathrm{e}}$ année du primaire. Ces jeunes semblent également plus à risque d'être victime d'intimidation de la part de leurs pairs et c'est justement la dimension des relations avec les pairs, soit le soutien qu'ils perçoivent de leurs pairs qui semble l'aspect du fonctionnement interpersonnel qui s'associe à une plus grande vulnérabilité à la dépression à plus long terme, soit au cours de l'adolescence et des études secondaires. Ces jeunes garçons, et cela semble également être présent chez les filles, qui ont rapporté qu'ils bénéficiaient d'un grand soutien de leurs amis à la fin de l'enfance et de l'école primaire se révèlent ceux qui rapportent une augmentation ou une absence de diminution de leurs symptômes de dépression au cours du secondaire. On peut se demander si ces garçons vulnérables à 1112 ans, soit en $6^{e}$ année, non seulement parce qu'ils vivent une puberté précoce associée à une image corporelle négative, mais également parce qu'ils vivent des conflits familiaux, ne compensent pas 


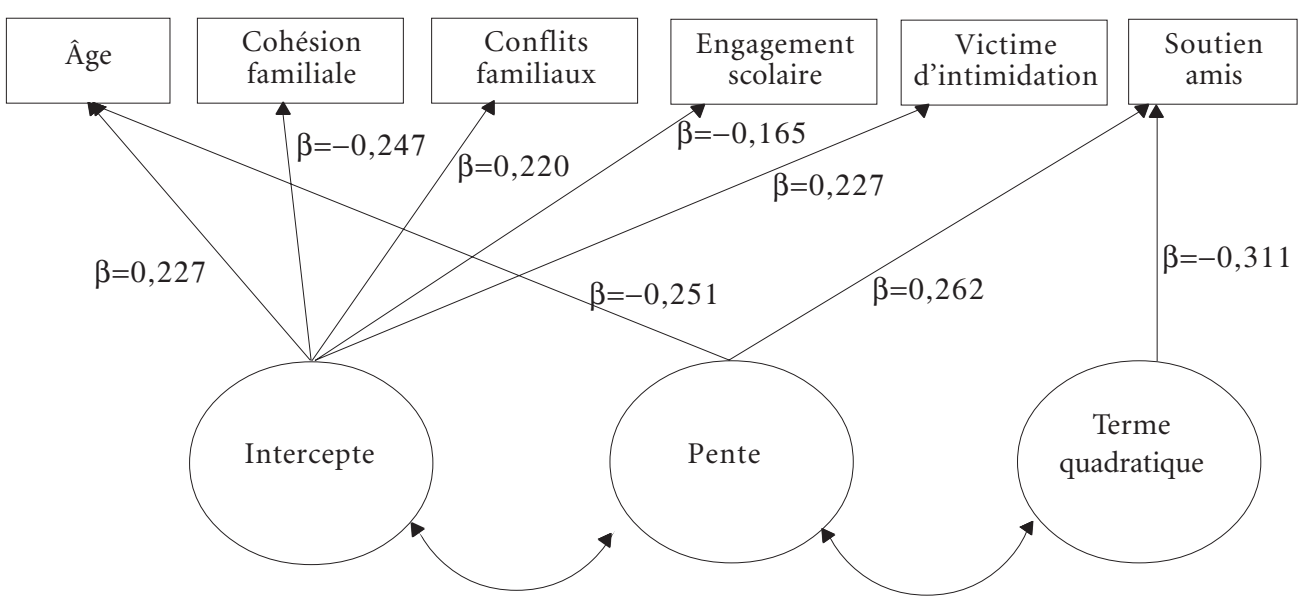

Figure 3. Description des effets (bêtas standardisés) des covariables significatives du modèle final des filles sur l'intercepte, la pente et le terme quadratique. Tous les effets sont significatifs à $p<0,05$.

par le soutien de quelques amis proches avec lesquels une certaine forme de dépendance affective serait présente. On pourrait penser que la perte de ce soutien lors de la transition vers le secondaire les rendrait plus vulnérables à la dépression.

Chez les filles, une image corporelle négative, davantage que le statut pubertaire, lorsqu'elle est associée à des difficultés familiales, se reflétant dans la présence de conflits familiaux et d'une faible cohésion familiale, les rendrait aussi plus dépendantes du soutien de leurs amis. Chez elles, on remarque également que l'engagement scolaire, soit le fait de participer aux activités de la classe, aux discussions et d'aimer l'école, de même que le rendement scolaire mais de façon plus marginale, sont associés à la présence de moins de symptômes de dépression, alors que la relation inverse est présente pour l'âge, que l'on peut interpréter comme la présence possible de redoublement dans le parcours scolaire.

Ces résultats nous apparaissent s'orienter dans le sens des modèles proposés par les auteurs tels que Eccles et al. ${ }^{35}$ et Simmons et al. ${ }^{36,37}$ qui soulignent l'effet négatif de la puberté précoce. Nos résultats proposent cependant que ce soit possiblement lorsque cette dernière est associée à une image corporelle négative, qu'une vulnérabilité à la dépression pourrait apparaître. Également, l'importance du réseau de soutien des pairs ainsi que le risque associé à l'interruption du développement de ce réseau ou à la perte des amis lors de la transition scolaire qui se produit en synchronie avec le passage de l'enfance à l'adolescence, est aussi un élément des modèles présentés dans les écrits qui est confirmé par les résultats de notre étude, le soutien des pairs ressortant d'ailleurs comme l'une des pierres angulaires possiblement de premiers ordres dans la réussite de cette transition en ce qui a trait à l'émergence de la dépression. De nouvelles analyses seront cependant nécessaires afin d'identifier l'existence possible de sous-groupes de jeunes présentant des trajectoires distinctes au niveau des symptômes dépressifs, de même que les variables qui seraient associées à chacune de ces trajectoires.

Ces résultats soulignent l'importance d'intervenir de façon préventive auprès des jeunes adolescents à risque afin de faciliter le passage vers cette étape de la vie. À cet effet, nous avons développé un programme de prévention de la dépression s'adressant spécifiquement à la population adolescente. Le programme Pare-Chocs ${ }^{38}$ est un programme de prévention ciblée de type indiquée ou sélective s'adressant aux adolescents de 14 à 17 ans ainsi qu'à leurs parents. Il peut être utilisé auprès de jeunes présentant des symptômes de dépression (type indiqué) ou des facteurs de risque de dépression (ex : la présence d'un parent dépressif, le divorce des parents, de faibles habiletés sociales ou de communication) (type sélectif). Animé par deux intervenants, Pare-Chocs comprend 55 activités réparties en douze rencontres avec des groupes de six à dix adolescents. Trois rencontres-parents sont également offer- 
tes. En lien avec les résultats de cette étude suggérant l'importance du soutien social, ce programme comprend des composantes visant le développement d'habiletés sociales, soit des habiletés de communication, de négociation et de résolution de problèmes interpersonnels. Également en lien avec nos résultats, une composante vise à aider les adolescents à développer une image corporelle et une estime de soi positives. Utilisant une approche cognitivo-comportementale, d'autres composantes du programme incluent la restructuration cognitive, le développement d'habiletés d'auto-observation et l'augmentation des activités plaisantes, l'apprentissage de techniques de relaxation et le développement des connaissances sur la dépression. Une composante familiale est également incluse dans Pare-Chocs et trois rencontres sont offertes aux parents en parallèle des rencontres offertes aux adolescents. Ce programme a fait l'objet d'études évaluatives qui soutiennent son efficacité ${ }^{39-41}$.

En terminant, il s'avère important de nommer certaines limites de cette étude. Notamment, les mesures ont été recueillies à chaque année en début d'année scolaire. Des mesures répétées en début et fin d'année aurait permis de mesurer les changements de façon plus précises suite à la transition. De plus, cette étude mériterait d'être répliquée auprès d'un échantillon répondant à un diagnostic de dépression majeure afin de comparer des échantillons cliniques et populationnels. Finalement, certains chercheurs considèrent la mesure du temps comme une variable discrète plutôt que continue ce qui peut influencer l'interprétation des coefficients bêta.

En conclusion, les résultats de cette étude démontrent que la période de transition entre l'enfance et l'adolescence s'avère une étape de vulnérabilité à la dépression chez les garçons et les filles qui méritent d'apporter une attention particulière aux programmes de prévention en santé mentale chez cette population. Malgré que des stratégies d'intervention se soient développées au cours des dernières années, il demeure que trop peu d'adolescents à risque reçoivent un traitement afin de les aider à traverser cette étape majeure de la vie. Les études démontrent d'ailleurs que seulement $25 \%$ des adolescents dépressifs reçoivent une intervention ${ }^{42}$. Le programme Pare-Chocs a démontré son efficacité à réduire les symptômes de dépression chez les adolescents et constitue une stratégie d'intervention préventive intéressante.

\section{Collaborations}

D Marcotte et A Lemieux ont participé à toutes les étapes de elaboration de l'article. 


\section{Références}

1. Costello EJ, Angold A, Burns BJ, Stangl DK, Tweed DL, Erkanli A, Worthman CM. The great smoky mountains study of youths: goals, design, methods, and the prevalence of DSM-III-R disorders. Arch Gen Psychiatry 1996; 53(12):1129-1136.

2. Birmaher B, Arbelaez C, Brent D. Course and outcome of child and adolescent major depressive. Course and outcome of child and adolescent major depressive disorder. Child Adolesc Psychiatr Clin N Am 2002; 11(3):619-637.

3. Kovacs M, Feinberg TL, Crouse-Novak M, Paulauskas SL, Pollock M, Finkelstein R. Depressive disorders in childhood: II. A longitudinal study of the risk for a subsequent major depression. Arch Gen Psychiatry 1984; 41(7):643-649

4. Lewinsohn PM, Hops H, Roberts RE, Seeley JR, Andrews JA. Adolescent psychopathology: I. Prevalence and incidence of depression and other DSMIII-R disorders in high school students. J Abnorm Psychol 1993; 102(1):133-144.

5. Kim-Cohen J, Caspi A, Moffitt TE, Harrington $\mathrm{H}$, Milne BJ, Poulton R. Prior juvenile diagnoses in adults with mental disorder: Developmental follow-back of a prospective-longitudinal cohort. Arch Gen Psychiatry 2003; 60(7):709-717.

6. McGee R, Feehan M, Williams S, Anderson J. DSMIII disorders from age 11 to age 15 years. J Am Acad Child Adolesc Psychiatry 1992; 31(1):50-59.

7. Angold A, Costello EJ, Worthman CM. Puberty and depression: The roles of age, pubertal status and pubertal timing. Psychol Med 1998; 28(1):51-61.

8. Kessler RC, Zhao S, Blazer DG, Swartz M. Prevalence, correlates, and course of minor depression and major depression in the national comorbidity survey. J Affect Disord 1997; 45(1-2):19-30.

9. Hankin BL, Abramson LY, Moffitt TE, Silva PA McGee R, Angell KE. Development of depression from a preadolescence to young adulthood: Emerging gender differences in a 10-year longitudinal study. Journal of Abnormal Psychology 1998; 107(1): 128-140.

10. Petersen AC, Sarigiani PA, Kennedy RE. Adolescent depression: Why more girls? J Youth Adolesc 1991; 20(2):247-271.

11. González-Tejera G, Canino G, Ramírez R, Chávez L, Shrout P, Bird H, Bravo M, Martínez-Taboas A, Ribera J, Bauermeister J. Examining minor depression and major depression in adolescents. J Child Psychol Psychiatry 2005; 46(8):888-899.

12. Twenge JM, Nolen-Hoeksema S. Age, gender, race, socioeconomic status, and birth cohort differences on the Children's Depression Inventory: A metaanalysis. J Abnorm Psychol 2002; 111(4):578-588.

13. Dekker MC, Ferdinand RF, van Lang ND, Bongers IL, van der Ende J, Verhulst FC. Developmental trajectories of depressive symptoms from early childhood to late adolescence: Gender differences and adult outcome. J Child Psychol Psychiatry 2007; 48(7):657-666.

14. Brendgen M, Wanner B, Morin AJ, Vitaro F. Relations with parents and with peers, temperament, and trajectories of depressed mood during early adolescence. J Abnorm Child Psychol 2005; 33(5):579-594.
15. Wang M-T, Brinkworth M, Eccles J. Moderating Effects of Teacher-Student Relationship in Adolescent Trajectories of Emotional and Behavioral Adjustment. Dev Psychol 2013; 49(4):690-705.

16. Québec. Ministère de l'Éducation du Loisir et du Sport. Indicateurs de l'éducation. Québec: Gouvernement du Québec; 2008.

17. Beck AT, Steer R, Brown GK. IDB-II. Inventaire de Dépression de Beck. Paris: Les Éditions du Centre de psychologie appliquée; 1998.

18. Weissman A, Beck AT. Development and validation of the Dysfunctional Attitude Scale. Paper presented at the meeting of the Association for Advancement of Behavior Therapy, Chicago 1978.

19. Power MJ, Katz R, McGuffin P, Duggan CF, Lam D, Beck AT. The Dysfunctional Attitude Scale (DAS). A comparison of forms A and B and proposals fora new subscaled version. Journal of Research in Personality 1994; 28(3):263-276.

20. Petersen AC, Crockett L, Richards M, Boxer A. A self-report measure of pubertal status: Reliability, validity, and initial norms. J Youth Adolesc 1988; 17(2):117-133.

21. Verlaan P, Cantin S, Boivin M. Validation de l'échelle de développement physique: Évaluation du niveau de maturation pubertaire à l'adolescence, Revue canadienne des sciences du comportement 2001; 33(3):143-147.

22. Harter S. Manual for the Self-Perception Profile for Adolescents. Denver: University of Denver Press; 1988.

23. Procidano ME, Heller K. Measures of Perceived Social Support from Friends and from Family: Three Validation Studies. Am J Community Psychol 1983; 11(1):1-24

24. Olweus D. Bullying at school: basic facts and effects of a school based intervention program. J Child Psychol Psychiatry 1994; 35(7):1171-1190.

25. Moos RH, Tricket EJ. Manual for the Family Environment Scale. Palo Alto: Consulting Psychologists Press; 1981

26. Moos RH, Tricket EJ. Classroom Environment Scale Manual. $2^{\text {nd }}$ Edition. Palo Alto: Consulting Psychologists Press; 1987.

27. Potvin P, Doré-Côté A, Fortin L, Royer É, Marcotte D, Leclerc D. Questionnaire d'identification des élèves à risque de décrochage scolaire (logiciel). Québec: CTREQ éditeur; 2007.

28. Reynolds CR, Kamphaus RW. Behavior Assessment System for Children. Circle Pines: American Guidance Service; 1998.

29. Reynolds CR, Kamphaus RW. The Clinician Guide to The Behavior Assessment System for Children (BASC). New York: Guilford Press; 2002.

30. Frick PJ. Conduct Disorders and Severe Antisocial Behavior. New York: Plenum Press; 1998.

31. Brown MW, Cudeck R. Alternative ways of assessing model fit. In: Bollen KA, Long JS, editors. Testing structural equation models. Newbury Park, CA: Sage; 1993. p. 136-162.

32. Bentler PM. Comparative fit indexes in structural models. Psychological Bulletin 1990; 107(2):238-246. 
33. Hu Li-tze, Bentler PM. Cutoff criteria for fit indexes in covariance structure analysis: Conventional criteria versus new alternatives. Structural Equation Modeling 1999; 6(1):1-55.

34. Bollen KA. Structural Equations with Latent Variables. New York: John Wiley \& Sons; 1989.

35. Eccles JS, Lord S, Buchanan CM. School transitions in early adolescence: What are we doing to our young people? Dans: Graber JA, Brooks-Gunn J, Petersen AC, editors. Transitions through Adolescence: Interpersonal Domains and Context. Hillsdale: Lawrence Erlbaum Associates; 1996. p. 251-284.

36. Simmons RG, Blyth DA. Moving into Adolescence: The Impact of Pubertal Change and School Context. New York: A. de Gruyter; 1987.

37. Simmons RG, Blyth D, Van Cleave EF, Bush DM. Entry into early adolescence: The impact of school structure, puberty and early dating on self-esteem. Am Sociol Rev 1979; 44(6):948-967.

38. Marcotte D. PARE-CHOCS, programme d'intervention auprès d'adolescents dépressifs. Manuel de l'animateur et Cahier du participant. Québec: Septembre éditeur; 2006.

39. Poirier M, Marcotte D, Joly J, Fortin L. Program and implementation effects of a cognitive-behavioral intervention for preventing depression among adolescents at-risk of school dropout, depressive type. $E d-$ ucational Research and Evaluation. In press 2013.

40. Poirier M, Marcotte D, Joly J, Fortin L. Évaluation de la qualité de l'implantation du programme PareChocs en contexte scolaire. Revue pour la recherche en éducation. Sous presse 2013.

41. Mesquida L, Arff A, Marcotte D, Raynaud JP. Depression in adolescence: applying a school-based prevention program in France. Poster presented at the annual congress of International association for child and adolescent psychiatry and allied professions (IACAPAP). Paris: Juillet; 2012.

42. Beardslee WR, Salt P, Porterfield K, Rothberg PC, van de Velde P, Swatling S, Hoke L, Moilanen DL, Wheelock I. Comparison of preventive interventions for families with parental affective disorder. $J \mathrm{Am}$ Acad Child Adolesc Psychiatry 1993; 32(2):254-263.

Artigo apresentado em 04/09/2013

Aprovado em 26/10/2013

Versão final apresentada em 05/11/2013 